\title{
Quantum creep in layered antiferromagnetic superconductor
}

Tomasz Krzysztońa

Institute of Low Temperature and Structure Research, Polish Academy of Sciences, 50-950 Wrocław, P.O. Box 1410, Poland

Received 20 September 2012 / Received in final form 22 November 2012

Published online 21 January 2013

(c) The Author(s) 2013. This article is published with open access at Springerlink.com

\begin{abstract}
In the mixed state of layered superconductor, the antiferromagnetic order of magnetic ions can create the spin-flop domains along the phase cores of the Josephson vortices, and this property impact upon the creep rate in the antiferromagnetic superconductor. The activation of the creep at constant temperature can be either thermal or quantum, depending on the intensity, or direction of the applied magnetic field. It is also shown that the action, and hence the activation energy, is rendered temperature dependent, when the damping and inertial mass of the vortex are included, so that the quantum tunnelling rate becomes temperature dependent below the crossover temperature.
\end{abstract}

\section{Introduction}

The discoveries of ternary rare earth (RE) Chevrel Phases $\mathrm{REMo}_{6} \mathrm{~S}_{8}$ and $\mathrm{RERh}_{4} \mathrm{~B}_{4}[1,2]$ compounds with regular distribution of localized magnetic moments of $\mathrm{RE}$ atoms have proved conclusively the coexistence of various types of magnetism with superconductivity. Intensive experimental and theoretical research has shown that $4 f$ electrons of $\mathrm{RE}$ atoms responsible for magnetism and $4 d$ electrons of molybdenum chalcogenide or rhodium boride clusters responsible for superconductivity are spatially separated and therefore their interaction is weak. In many of these systems, superconductivity coexists rather easily with antiferromagnetic order, where usually the Néel temperature $T_{N}$ is lower than the critical temperature for superconductivity $T_{c}$. For almost two decades the problem of the interaction between magnetism and superconductivity has been overshadowed by high temperature superconductivity (HTS) found in copper oxides. However, the discovery of the magnetic order in Ru-based superconductors $[3,4]$ inspired a return to the so-called coexistence phenomenon [5]. The interplay between magnetism and superconductivity was studied in $d$-electron $\mathrm{UGe}_{2}$ [6] and $\mathrm{ZrZn}_{2}$ [7], where itinerant ferromagnetism may coexist with superconductivity, and in heavy fermion $\mathrm{UPd}_{2} \mathrm{Al}_{3}$ [8], where magnetic excitons are present in the superconducting phase. The recent discovery of the iron pnictide superconductors [9] have triggered broad interest in the mechanism of the coexistence of magnetism and superconductivity in this new class of superconductors [10]. They exhibit qualitative similarity to cuprates in that superconductivity occurs upon carrier doping (elec-

\footnotetext{
a e-mail: tomaszkrzyszton@gmail.com
}

trons, in this case) of pristine compounds that exhibit magnetism [11].

Among classic magnetic superconductors, the Chevrel phases have been studied most intensively. These compounds are mainly polycrystalline materials. However, some specific effects can be measured only on single crystals. One such effect is a two-step flux penetration process, predicted in references $[12,13]$ and after that discovered in the antiferromagnetic superconductor (bct) $\mathrm{ErRh}_{4} \mathrm{~B}_{4}$ [14]. Later it was observed also in $\mathrm{DyMo}_{6} \mathrm{~S}_{8}[15]$ that specific effect is a consequence of creation of the spin-flop (or metamagnetic) domain along the vortex core.

Consider, as an example, antiferromagnet with two magnetic sublattices. An infinitesimal magnetic field applied perpendicular to its easy axis makes the ground state unstable against the phase transformation to the canted phase (spin-flop). On the other hand, if the magnetic field is applied parallel to the easy axis, the antiferromagnetic configuration is stable up to the thermodynamic critical field $H_{T}$. When the field is further increased a canted phase develops in the system. Assume that in the antiferromagnetic superconductor the lower critical field fulfils the relation $H_{c 1}<\frac{1}{2} H_{T}$ and that the external field, $H_{c 1}<H<\frac{1}{2} H_{T}$, is applied parallel to the easy axis. Then the superconducting vortices appear in the ground antiferromagnetic state. If the field is increased above $\frac{1}{2} H_{T}$ in the core originates the phase transition to the canted phase because the field intensity in the core doubles the intensity of the external field [16]. The spatial distribution of the magnetic field of the vortex is a decreasing function of the distance from the center of the vortex. Hence the magnetic field intensity in the neighborhood of the core is less than $H_{T}$. Therefore, the rest of the vortex remains 


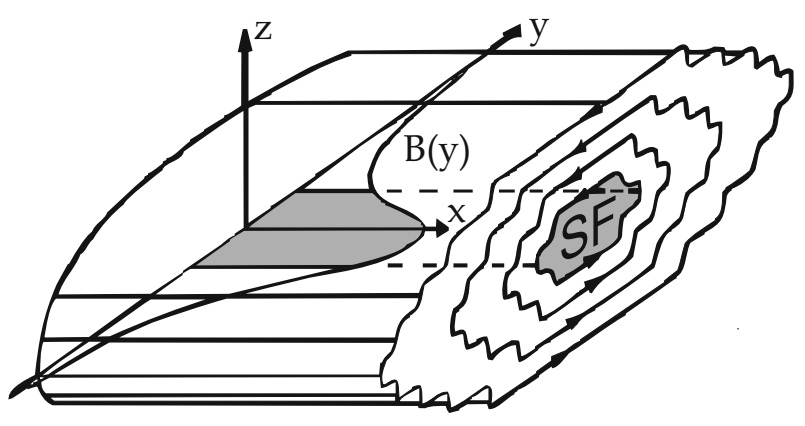

Fig. 1. Single Josephson vortex lying in the ab plane along the $\hat{x}$-axis ( $\hat{a}$-axis). The SF domain induced along the phase core is shown in the gray area.

in the antiferromagnetic configuration. The radius of spin flop domain grows as the external field is increased.

Thus, in the considered model there are two distinct types of vortices. Possible candidate of such system might be $\mathrm{ErBa}_{2} \mathrm{Cu}_{3} \mathrm{O}_{7}$, however, the features described in this paper have not yet been experimentally investigated. That compound has tetragonal unit cell with small orthorombic distortion in the ab plane. The Er ions form two sublattices antiferromagnetic structure of magnetic moments laying parallel and antiparallel to the a direction [17]. We shall use in this paper an abstract structure which resembles the above mentioned structure of $\mathrm{ErBa}_{2} \mathrm{Cu}_{3} \mathrm{O}_{7}$. It consists of the superconducting layers of thickness $d_{s}$ and the isolating layers of thickness $d_{i}, d=d_{i}+d_{s}$. In the isolating layers, the magnetic moments are running parallel and antiparallel to the a direction (easy axis). The magnetic field aligned parallel to the conducting planes makes the vortex lattice accommodate itself to the layer structure so that the vortex cores are lying between the superconducting sheets.

The structure of a vortex lying in the ab plane in a layered superconductor with Josephson coupling between adjacent layers resembles the Abrikosov's one except that the order parameter does not vanish anywhere [16]. Instead there exists a region where the Josephson current $j_{z}$ is of the order of the critical current. In this region, named the phase core, the London model fails as in the classic superconductor. Away from the phase core the streamlines of the shielding supercurrents, which also represents contours of constant magnetic field, are elliptical except for the zigzags, shown in Figure 1, due to the intervening insulating layers.

\section{Macroscopic quantum tunnelling through intrinsic pinning barriers}

A current density $j$, flowing along the planes perpendicular to the applied magnetic field exerts a Lorentz force on the vortices in the $\mathbf{c}$ direction so that intrinsic pinning barriers are formed on superconducting layers. The experimental evidence of quantum tunnelling is based on the fact that the magnetic moment relaxation rate exhibits two types of behavior as a function of temperature. Above a characteristic temperature $T_{0}$ in the thermal activation regime the decay rate is of the Arrhenius type $\Gamma \sim \exp \left(-U_{0} / k_{B} T\right)$. Below $T_{0}$, the decay rate is assumed a priori to be essentially independent of temperature $\Gamma \sim \exp (-S / \hbar)$ and is interpreted as arising from the quantum tunneling of vortices through intrinsic pinning potential [18-20]. In the following, we show a considerable change of tunneling rate and crossover temperature due to the SF phase formation in the vortex core. We shall assume that the vortex line is a straight stringlike object, with effective mass $m$ per unit length, moving in a metastable intrinsic pinning potential $V(u)$ and exposed to continuous deformation $u(x, t)$ in the $\hat{z}$ direction. The magnetic field is applied in $\hat{x}$ direction. In the semiclassical approximation the quantum decay rate is calculated as a saddle-point solution (bounce) of the Euclidean action $S$ for the string. The present method improves calculation of [21] by including inertial mass of the vortex.

$$
\begin{aligned}
S= & \int_{-\infty}^{\infty} d x \int_{0}^{\hbar \beta} d \tau\left\{\frac{1}{2} m\left(\frac{\partial u}{\partial \tau}\right)^{2}+\frac{\varepsilon_{l}}{2}\left(\frac{\partial u}{\partial x}\right)^{2}+V(u)\right. \\
& \left.-\frac{\eta}{2 \pi} \frac{\partial u}{\partial \tau} \int_{0}^{\hbar \beta} d \tau^{\prime} \frac{\partial u}{\partial \tau^{\prime}} \ln \left|\sin \frac{\pi}{\hbar \beta}\left(\tau-\tau^{\prime}\right)\right|\right\}
\end{aligned}
$$

Here $\beta=\left(k_{B} T\right)^{-1}, \eta$ is the viscosity coefficient and $\tau$ denotes imaginary time. The pinning potential $V(u)$ consists of intrinsic periodic part and the Lorentz potential:

$$
V(u)=-\frac{\varphi_{0} j_{c} d}{2 \pi} \cos \left(\frac{2 \pi u}{d}\right)-\varphi_{0} j u,
$$

where $j_{c}$ denotes critical depinning current. For the large current, this potential can be expanded around the inflection point to give:

$$
V(u)=V_{0}\left[\left(\frac{u}{w}\right)^{2}-\left(\frac{u}{w}\right)^{3}\right]
$$

where $V_{0}=\frac{2}{3} \frac{\varphi_{0} j_{c} \pi^{2}}{d^{2}} w^{3}$ and $w=\frac{3 d}{\pi}\left(\frac{j_{c}-j}{2 j_{c}}\right)^{\frac{1}{2}}$ may be thought as the height and width of the barrier (because $V(0)=V(w)=0)$, and $j_{c}$ is the critical depinning current. The last term in equation (1), so called CaldeiraLeggett [22] action, describes the ohmic damping produced by the coupling to the heat-bath reservoir of harmonic oscillators interacting linearly with the vortex. The line tension $\varepsilon_{l}$ is different for vortices in two different orientations ( $\mathbf{a}$ and $\mathbf{b}$ ) in the $\mathbf{a b}$ plane. The Euler-Lagrange equation of motion [23] on semiclassical trajectory is:

$m \frac{\partial^{2} u}{\partial \tau^{2}}+\varepsilon_{l} \frac{\partial^{2} u}{\partial x^{2}}-V^{\prime}(u)-\frac{\eta}{\hbar \beta} \int_{0}^{\hbar \beta} d \tau \frac{\partial u}{\partial \tau} \cot \frac{\pi}{\hbar \beta}\left(\tau-\tau^{\prime}\right)=0$.

In the thermal regime $T>T_{c r}$, the classical trajectory $u_{0}(x)$ gives the activation energy $U_{0}$ from the static solution of the following equation:

$$
-\varepsilon_{l} \frac{\partial^{2} u_{n}}{\partial x^{2}}+V^{\prime}\left(u_{0}\right)=0
$$


Below the crossover temperature $T<T_{c r}$, a new kind of trajectory, periodic in the imaginary time, develops. Therefore, $u(x, \tau)$ can be expanded in the Fourier series with Matsubara frequencies

$$
u(x, \tau)=\sum_{n=0}^{\infty} u_{n}(x) \cos \left(\omega_{n} \tau\right) ; \quad \omega_{n}=\frac{2 \pi n}{\hbar \beta} .
$$

Substituting this expansion into equation (3) and linearizing potential around the static solution $u_{0}(x)$ one obtains:

$$
-\varepsilon_{l} \frac{\partial^{2} u_{n}}{\partial x^{2}}+V^{\prime \prime}\left(u_{0}\right) u_{n}=-\left(\eta \omega_{n}-m \omega_{n}^{2}\right) u_{n} .
$$

Upon introducing new variables $v_{n}=\frac{u_{n}}{w}$ and $\zeta=$ $\frac{x}{d}\left(\frac{\pi^{2} w \varphi_{0} j_{c}}{\varepsilon_{l}}\right)^{\frac{1}{2}}$ the static equation now reads:

$$
-\frac{1}{2} \frac{\partial^{2} v_{0}}{\partial \zeta^{2}}+2 v_{0}-3 v_{0}^{2}=0 .
$$

The above nonlinear equation has been solved in [24], and also in [25], to give:

$$
v_{0}=\cosh ^{-2} \zeta
$$

Substitution of equation (6) into equation (4) results in the following equation:

$$
-\frac{1}{2} \frac{\partial^{2} v_{n}}{\partial \zeta^{2}}+2\left(1-3 \cosh ^{-2} \zeta\right) v_{n}=E_{n} v_{n}
$$

where

$$
E_{n}=-\frac{j_{c} w^{2}}{V_{0}^{2}}\left(\eta \omega_{n}+m \omega_{n}^{2}\right)
$$

Equation (10) has three discrete eigenvalues: $-\frac{5}{2}, 0, \frac{3}{2}$. The negative one determines the crossover temperature:

$$
k_{B} T_{c r}=\frac{\hbar \eta}{4 \pi m}\left(\sqrt{1+14.2 \frac{\pi \varphi_{0} j_{c} m}{d \eta^{2}} \sqrt{1-\frac{j}{j_{c}}}}-1\right) .
$$

In the thermally activated region above $T_{c r}$ we use the static solution of equation (5) and get the action $S_{0}=U_{0} \hbar \beta$ which arises only from the elastic and pinning terms of the Euclidean action (Eq. (1)). Now the action $S_{0}$ reads:

$$
S_{0}=2.77\left(\frac{\varepsilon_{l} d^{3} \varphi_{0} j_{c}}{\pi^{3}}\right)^{\frac{1}{2}}\left(1-\frac{j}{j_{c}}\right)^{5 / 4} \hbar \beta .
$$

Below $T_{c r}$, the predominant mechanism of the decay of meta-stable state is the quantum-mechanical tunneling, and the action on the bounce trajectory determines the rate of this process. To include contributions of effective mass and viscous damping in the bounce action it is necessary to go beyond the harmonic approximation in equation $(7)$ and substitute for $u(x, \tau)$ the leading terms in the Matsubara frequencies [26]. Calculations are performed in the vicinity of crossover temperature so the perturbation parameter is $\left(1-T / T_{c r}\right)$. It can be shown that $v_{n}$ are of the order $\left(1-T / T_{c r}\right)^{\frac{n}{2}}$ and the leading-order contribution comes from $v_{1} \sim \cosh ^{-3} \zeta$. Substituting this term for $u$ in equation (1), one can obtain complete action for the bounce trajectory involving contribution of the inertial mass and the viscous damping.

$$
\begin{aligned}
S= & 5.26 \hbar \beta\left[\frac{\varepsilon_{l} d^{3} \varphi_{0} j_{c}}{\pi}\right]^{1 / 2}\left(1-\frac{j}{j_{c}}\right)^{5 / 4} \\
& +\left[\frac{\varepsilon_{l} d^{5}}{\pi \varphi_{0} j_{c}}\right]^{1 / 2}\left(5.71 \frac{m}{\hbar \beta}+2.85 \frac{\eta}{\pi}\right)\left(1-\frac{j}{j_{c}}\right)^{3 / 4} .
\end{aligned}
$$

It is interesting to compare the present results with those obtained previously by Smith [27], who used variational ansatz in order to solve relaxation of a vortex trapped into metastable state. In that work, the crossover temperature calculated in the limit of large driving current shows the pretty good numerical agreement with the equation (12). When the mass of the vortex is neglected, the result obtained here for $S_{0}$ is similar to the one of Smith for the Euclidean action on thermally activated trajectory. However, the complete action of equation (14), calculated in the vicinity of crossover temperature via perturbation method, can not be applied to $T=0$ domain.

\section{Evaluation of the parameters}

The above calculations apply to both kind of vortices characterized by different effective masses, line tensions and viscosity coefficients. We shall calculate these parameters as functions of condensation energy accumulated in the vortex cores. The detailed analysis of this effect was given in the work of Bardeen and Stephen [28]. We shall follow their argumentation but without going into details.

For the stationary flux flow the viscous force $\eta \frac{\partial u}{\partial t}$ is equal to Lorentz force. The electric field generated by the moving vortex is $E=B \frac{\partial u}{\partial t}$, so we get $E=\frac{\varphi_{0} B}{\eta} j=\rho j=$ $\rho_{N} \frac{B}{H_{c 2}} j$ where $\rho_{N}$ is the normal phase resistivity in the ab plane and $H_{c 2}$ is the upper critical field parallel to the layers. Finally,

$$
\eta=\frac{\varphi_{0} H_{c 2}}{\rho_{N}}=\frac{\varphi_{0} \kappa H_{c} \sqrt{2}}{\rho_{N}}=\varepsilon_{l} \frac{4 \sqrt{3} \kappa^{2}}{\pi \rho_{N} \ln \kappa},
$$

where $H_{c}=\frac{\varepsilon_{l} \kappa 2 \sqrt{6}}{\pi \varphi_{0} \ln \kappa}$ is calculated from the constitutive relation $\varepsilon_{l}=H_{c 1} \varphi_{0}$.

A moving vortex in the magnetic superconductor can transfer energy to the magnetic moments by emitting spin waves. This energy transfer gives rise to magnetic contribution to the mass and viscosity of the vortex [29]. However the vortex velocity for this effect to occur should exceed the velocity of the spin wave which is not the case during the flux creep.

The effective mass of the vortex can be deduced from the work of Suhl [30]. He derived the core contribution 
to the inertial mass $m=\frac{3}{8} m_{e} \frac{\xi^{2} H_{c}^{2} \mu_{0}}{\epsilon_{F}}$, where $m_{e}$ denotes the mass of the electron and $\epsilon_{F}$ the Fermi energy, and the electromagnetic contribution coming from the energy of the electric field induced by the moving flux. Simple estimation shows that this contribution in layered superconductors is $10^{-4}$ of the core contribution. Therefore,

$$
m=\varepsilon_{l}^{2} \frac{9 \lambda_{a b}^{2} m_{e} \mu_{0}}{\varphi_{0}^{2} \pi^{2} \epsilon_{F}(\ln \kappa)^{2}} .
$$

The vortices in two main orientations in the ab plane have different line tensions. These lying parallel to $\mathbf{b}$ direction, and these laying in the a direction, but created in the magnetic field less than $\frac{1}{2} H_{T}$, have the line tension $\varepsilon_{b}$, and the ones lying in the a direction, but possessing SF domain inside, have the line tension $\varepsilon_{a}$.

$\varepsilon_{b}=\epsilon_{0} \ln \frac{\lambda_{a b}}{d} ; \varepsilon_{a}=\frac{\varphi_{0} H_{T}}{2}+\frac{9}{128} \epsilon_{0} \ln \frac{\varphi_{0} \lambda_{c}^{2}}{\pi\left(\mu_{0} H_{T}+M\right) d^{2} \lambda_{a b}^{2}}$,

where $\epsilon_{0}=\frac{\varphi_{0}^{2}}{4 \pi \lambda_{a b} \lambda_{c} \mu_{0}}, M$ is the magnetic moment of the SF domain of the vortex and $H_{T}$ is the thermodynamic critical field for the spin-flop transformation. In order to evaluate the above line tensions it is necessary to calculate $H_{T}$ and $M$. Therefore, the following argumentation is proposed. At low fields, in the vicinity of the lower critical field $H_{c 1}$, the intensity of the field in the vortex core is $2 H_{c 1}$ [16]. When the external field is increased, the intensity of the magnetic field in the vortex core increases due to the superposition of the fields of the surrounding vortices. The field intensity in the core must reach $H_{T}$ in order to originate a transition to the SF phase. Thus, taking into account only $z$ nearest neighbors, we can write for the nonunilateral triangular lattice

$$
\begin{aligned}
\varphi_{0} H_{T}= & 2 \varphi_{0} H_{c 1}+4 z \varepsilon_{b}\left(\ln \frac{\lambda_{a b}}{d}\right)^{-1} \\
& \times\left[K_{0}\left(\frac{c}{\lambda_{a b}}\right)+2 K_{0}\left(\frac{c}{2 \lambda_{a b}} \sqrt{\frac{3 \lambda_{c}}{\lambda_{a b}}}\right)\right] \\
= & 2 \varepsilon_{b}+o\left(\varepsilon_{b}\right),
\end{aligned}
$$

where $c$ is the lattice constant. Although there are no precise measurements of the spin-flop transition in the antiferromagnetic high temperature superconductors, we assume that $\mu_{0} H_{T} \approx 40 \mathrm{mT}$. The typical value of $4.2 \mu_{\mathrm{B}}$ per Er ion per unit cell in $\mathrm{ErBa}_{2} \mathrm{Cu}_{3} \mathrm{O}_{7}$ [31] gives $M \approx 0,35 \mathrm{~T}$ and $B_{T} \approx 0,40 \mathrm{~T}$. Since $\varphi_{0} H_{c 1}=\varepsilon_{b}$ and $d / \xi_{c} \approx 1$ we obtain:

$$
\frac{\varepsilon_{a}}{\varepsilon_{b}}=1+\frac{36}{128} \frac{\ln \left(\frac{\varphi_{0} \lambda_{c}^{2}}{\pi\left(\mu_{0} H_{T}+M\right) d^{2} \lambda_{a b}^{2}}\right)}{\ln \left(\frac{\lambda_{a b}}{d}\right)} \approx 1.7 .
$$

It is now possible to relate the viscosity coefficient equation (15) and the mass of the vortex equation (16) to its line tension equation (17).

$$
\frac{\eta_{a}}{\eta_{b}}=\frac{\varepsilon_{a}}{\varepsilon_{b}}
$$

and

$$
\frac{m_{a}}{m_{b}}=\left(\frac{\varepsilon_{a}}{\varepsilon_{b}}\right)^{2}
$$

With the use of a simple consideration we can estimate the change of $j_{c}$ due to the creation of spin-flop domain along the vortex

$$
j_{c} \varphi_{0} d \sim \frac{1}{2} \int d y d z C(y, z)\left(\frac{\partial u_{z}}{\partial z}\right)^{2}
$$

where the Fourier transform of the compression modulus is given by [32]:

$$
C\left(k_{y}, k_{z}\right)=\frac{B^{2}}{\mu_{0}\left(1+\lambda_{a b}^{2} k_{z}^{2}+\lambda_{c}^{2} k_{y}^{2}\right)} .
$$

By taking $d y d z \sim \varphi_{0} / B ; u_{z} \sim d ; \frac{\partial}{\partial z} \sim k_{z} \sim k_{y}\left(\lambda_{c} / \lambda_{a b}\right)$ the estimation of the integral gives:

$$
j_{c}=\frac{B d}{4 \lambda_{a b}^{2}} .
$$

In the a direction, however, we have an additional contribution from the magnetic domain $d y d z \sim 5 \varphi_{0} / 8 \pi B_{T}$, so we get:

$$
j_{c a}=j_{c}+\frac{5 d B_{T}}{128 \lambda_{a b}^{2}}
$$

and finally $j_{c a} \approx 3 j_{c b}$.

\section{Motion of the flux in the quantum regime}

Consider a hollow cylindrical sample of the radius $a$, and of the wall thickness $g \ll a$ (thin wall approximation). The sample is placed in a magnetic field directed parallel to the axis of the cylinder and along the superconducting layers. A trapped flux in the system is $\Phi \cong\left(B_{i n}-B_{\text {ex }}\right)$, where $B_{i n}$ denotes the field inside the hole of the sample and $B_{e x}$ outside the sample, respectively. The motion of the flux is triggered off by an activation process in which segments of the flux line tunnel through an intrinsic pinning potential to the neighboring interlayer spacing. By applying Faraday's law we can easily calculate the electric field in the sample due to the change of the trapped flux

$$
E=-\frac{1}{2} \mu_{0} a g j_{c} \frac{d\left(j / j_{c}\right)}{d t}
$$

which is equal to the mean electric field associated with the motion of vortices $E=\varphi_{0} W L g$, where $L$ is the length of the sample in the direction of the applied field, and $W$ is the activation probability per unit volume and unit time. Activation probability in the weak damping approximation [33] is equal to $\sqrt{\frac{30 S_{0}}{\pi \hbar}} \exp \left(-\frac{S}{\hbar}\right)$. Combining all above together we get:

$$
\Omega t=-\int_{x(0)}^{x(t)} \frac{\exp (S / \hbar)}{\sqrt{S_{0} / \hbar}} d x
$$

where $x=j / j_{c} ; \Omega=38.83\left(\frac{\varphi_{0}}{\mu_{0} j_{c}}\right)\left(\frac{L}{2 \pi a}\right)$, and $2 \pi a$ is the length of the sample along the flow of the current. 
Table 1. Input parameters [19,21,34-36].

\begin{tabular}{cc}
\hline$d=10^{-9} \mathrm{~m}$ & $\eta_{b}=10^{-8} \mathrm{~kg} /(\mathrm{s} \mathrm{m})$ \\
$j_{c b}=10^{11} \mathrm{~A} / \mathrm{m}^{2}$ & $\lambda_{a b}=160 \mathrm{~nm}$ \\
$m_{b}=10^{-22} \mathrm{~kg} / \mathrm{m}$ & $\lambda_{c}=800 \mathrm{~nm}$ \\
$\epsilon_{F}=0.1 \mathrm{eV}$ & $\mu_{0} H_{c 2}^{a b}=150 \mathrm{~T}$ \\
\hline
\end{tabular}

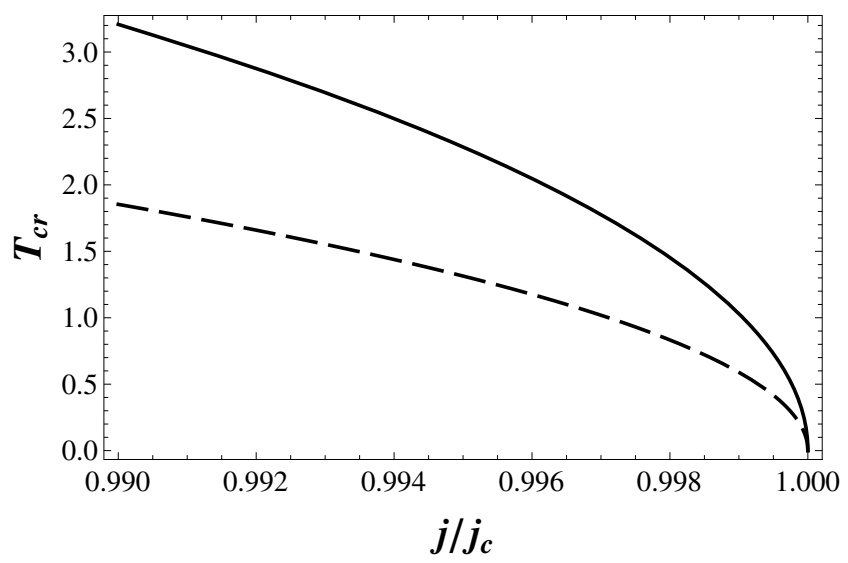

Fig. 2. Crossover temperature as a function of fractional current $j / j_{c}$. Dashed line depicts vortices possessing magnetic cores.

\section{Numerical results}

The purpose of the present study is to derive expressions for the bounce contribution to the action arising from the inertial mass term, elastic and pinning terms, and the damping term. As was previously mentioned, in the antiferromagnetic superconductors could occur vortices of two type. One type, quite similar to the vortices in nonmagnetic superconductor, and the other type possessing a ferromagnetic-like domain around and inside the core. Their line tension (Eq. (19)) and critical depinning current (Eq. (23)) is what distinguishes these vortices in the present study. The input parameters for numerical calculation are shown in Table 1.

We consider the limit of a large current, slightly less than critical depining current, and assume $\frac{L}{2 \pi a} \sim 1$. In this approximation we calculated the crossover temperature from equation (12) and plotted it as a function of (FC), fractional current $j / j_{c}$. As it can be seen, the difference of crossover temperatures for both types of vortices depends on the current, and for the value of $\mathrm{FC}$ equal to 0.99 this difference is about $1.3 \mathrm{~K}$, and vanishes when $\mathrm{FC}$ reaches 1 .

Figure 3 shows the activation energy $U_{0}$ calculated from equation (13) as a function of FC. The activation energy differs about $1.5 \mathrm{meV}$ for 0.99 of $\mathrm{FC}$ and goes to 0 when $\mathrm{FC}$ reaches 1.

Equation (24), solved numerically for the input parameters shown in the Table 1 , give the results shown on Figure 4. The results are consistent with the experimental findings for the YBCO class superconductors [36]. As we can see, the creep is slower when the vortices possessing magnetic domain occurred in the system. Another important effect is that the action and hence the activation energy is rendered temperature dependent so that the

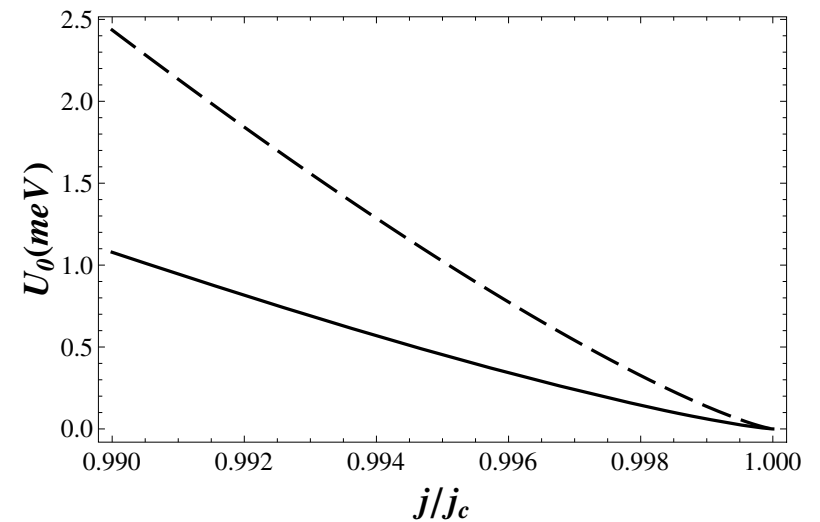

Fig. 3. Activation energy $U_{0}$ (in meV) calculated as a function of fractional current $j / j_{c}$. Dashed line depicts vortices possessing magnetic cores.

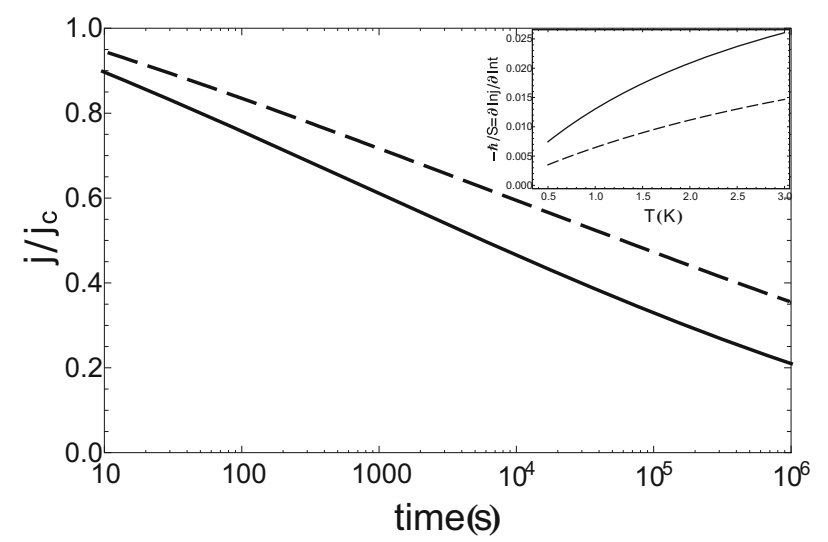

Fig. 4. Decay of the flux $\left(\Phi / \Phi_{0} \sim j / j_{c}\right)$ as a function of time (logarithmic scale), at fixed temperature $0.5 \mathrm{~K}$. In the inset, quantum creep rate as a function of temperature. Dashed line depicts vortices possessing magnetic cores.

quantum tunneling rate becomes temperature dependent below the crossover temperature.

\section{The "creep valve" mechanism}

There is another conclusion which follows from the above considerations. The creep regime can be altered even though the temperature is not changed. It is possible that the system leaps over from the quantum to the thermal creep regime, or vice versa, when the direction or the intensity of the applied magnetic field is changed at fixed temperature. To see how it can happen let us fix the temperature of the sample somewhere in the range $T_{c r b}>T_{0}>T_{\text {cra }}$, as shown in the Figure 5 , and increase the magnetic field intensity in the a direction to the point marked A. Now, the system is in the thermal creep regime. Then we change the direction of external field from the $\mathbf{a}$ to the $\mathbf{b}$ direction. The system now leaps over to the point B and finds itself in the quantum creep regime. Doing the same operation in the reverse order one enforces the system to crossover from the quantum to the thermal creep. The another scenario is also possible. When 


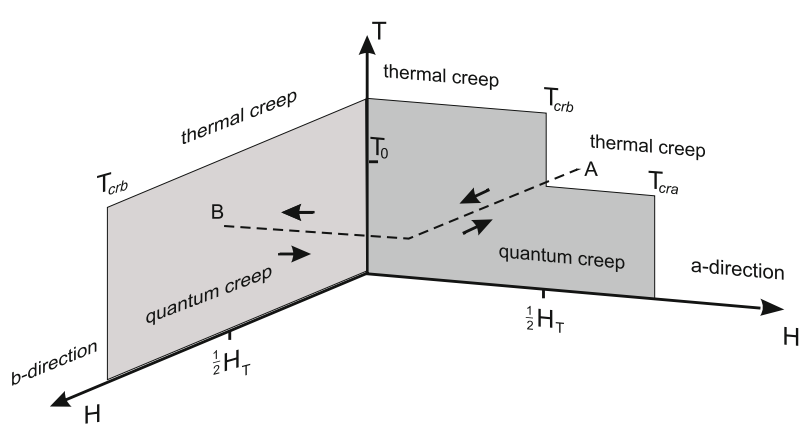

Fig. 5. Schematic diagram of the leap from thermal to quantum creep, and vice versa.

magnetic field is applied along the a direction and the temperature is fixed in the interval $T_{c r b}>T_{0}>T_{c r a}$, the increase of magnetic field intensity above $\frac{1}{2} H_{T}$ enforces the appearance of magnetic structure in the vortices, and changes the quantum activation to thermal one. Lowering the field intensity one can change back the creep from thermal to quantum regime. The described scenario could be named a "creep valve" because the creep rate can be decreased or increased by means of sweeping the field around the $\frac{1}{2} H_{T}$ value.

\section{Summary}

We discussed quantum tunneling of vortices in layered high temperature superconductor. When the damping and inertial mass of the vortex are included, the calculation shows that the activation energy is rendered temperature dependent so that the quantum tunnelling rate becomes temperature dependent below the crossover temperature. In the mixed state of the layered superconductor the antiferromagnetic order of magnetic ions can create the spinflop domains along the phase cores of the Josephson vortices, and this effect impact upon the creep rate in the antiferromagnetic superconductor. The activation of the creep at fixed temperature can be either thermal or quantum, depending on the intensity, or direction of the applied magnetic field.

\section{References}

1. M.B. Maple, H.C. Hamaker, L.D. Woolf, in Superconductivity in Ternary Compounds, edited by M.B. Maple, Ø. Fischer (Springer-Verlag, Berlin, 1982)

2. L.N. Bulaevskii, A.I. Buzdin, M. Kulić, S.V. Panjukov, Sov. Phys. Uspekhi 27, 927 (1984)

3. L. Bauernfeind, W. Widder, H.F. Braun, Physica C 254, $151(1995)$

4. D.J. Pringle, J.L. Tallon, B.G. Walker, H.J. Trodahl, Phys. Rev. B 59, R11679 (1999)

5. M.B. Maple, Physica B 215, 110 (1995)

6. S.S. Saxena, P. Agarwal, K. Ahilan, F.M. Grosche, R.K.W. Haselwimmer, M.J. Steiner, E. Pugh, I.R. Walker, S.R. Julian, P. Monthoux, G.G. Lonzarich, A. Huxley, I. Sheikin, D. Braithwaite, J. Flouquet, Nature 406, 587 (2000)
7. C. Pfleiderer, M. Uhlarz, S.M. Hayden, R. Vollmer, H.V. Lohneysen, N.R. Bernhoeft, G.G. Lonzarich, Nature 412, $58(2001)$

8. N.K. Sato, N. Aso, K. Miyake, R. Shiina, P. Thalmeier, G. Varelogiannis, C. Geibel, F. Steglich, P. Fulde, T. Komatsubara, Nature 410, 340 (2001)

9. Y. Kamihara, T. Watanabe, M. Hirano, H. Hosono, J. Am. Chem. Soc. 130, 3296 (2008)

10. S. Takeshita, R. Kadono, New J. Phys. 11, 035006 (2009)

11. Y. Nakai, K. Ishida, Y. Kamihara, M. Hirano, T. Matsui, H. Hosono, M. Seto, J. Phys. Soc. Jpn 77, 073701 (2008)

12. T. Krzysztoń, J. Magn. Magn. Mater. 15-18, 1572 (1980)

13. T. Krzysztoń, Phys. Lett. A 104, 225 (1984)

14. H. Iwasaki, M. Ikebe, Y. Muto, Phys. Rev. B 33, 4669 (1986)

15. T. Krzysztoń, K. Rogacki, Eur. Phys. J. B 30, 181 (2002)

16. J.R. Clem, M.W. Coffey, Phys. Rev. B 42, 6209 (1990)

17. T.W. Clinton, J.W. Lynn, J.Z. Liu, Y.X. Jia, R.N. Shelton, Physica C 217, 53 (1993)

18. G. Blatter, M.V. Feigel'man, V.B. Geshkenbein, A.I. Larkin, V.M. Vinokur, Rev. Mod. Phys. 66, 1125 (1994)

19. A.F.Th. Hoekstra, R. Griessen, A.M. Testa, J. el Fattahi, M. Brinkmann, K. Westerholt, W.K. Kwok, G.W. Crabtree, Phys. Rev. Lett. 80, 4293 (1999)

20. C.M. Smith, B. Ivlev, G. Blatter, Phys. Rev. B 52, 10581 (1995)

21. B.I. Ivlev, Yu.N. Ovchinnikov, R.S. Thompson, Phys. Rev. B 44, 7023 (1991)

22. A.O. Caldeira, A.J. Leggett, Ann. Phys. 149, 374 (1983)

23. H. Grabert, P. Olschowski, U. Weiss, Phys. Rev. B 36, 1931 (1987)

24. B.V. Petukhov, V.L. Pokrovsky, Zh. Eksp. Teor. Fiz. 63, 634 (1972) [Sov. Phys. JETP 36, 336 (1973)]

25. M. Büttiker, R. Landauer, Phys. Rev. A 23, 1397 (1981)

26. W.M. Gaber, B.N.N. Achar, Phys. Rev. B 52, 1314 (1995)

27. C.M. Smith, Europhys. Lett. 38, 551 (1997)

28. J. Bardeen, M.J. Stephen, Phys. Rev. 140, A1197 (1965)

29. A. Shekhter, L.N. Bulaevskii, C.D. Batista, Phys. Rev. Lett. 106, 037001 (2011)

30. H. Suhl, Phys. Rev. Lett. 14, 226 (1965)

31. Y. Abulafia, J. Barak, J.L. Peng, J. Appl. Phys. 76, 7468 (1994)

32. E.H. Brandt, Physica C 195, 1 (1992)

33. E. Šimánek, Inhomogeneous Superconductors. Granular and Quantum Effects (Oxford University Press, New York and Oxford, 1994), Chap. 4

34. D.R. Harshman, A.P. Mills Jr., Phys. Rev. B 45, 10684 (1992)

35. Y. Matsuda, N.P. Ong, Y.F. Yan, J.M. Harris, J.B. Peterson, Phys. Rev. B 49, 4380 (1994)

36. Sheng Luo, Chunguang Li, Yusheng He, Guohua Zhang, Duo Jin, Zhaoija Chen, Guangcheng Xiong, Guijun Lian, Daole Yin, C.E. Gough, Supercond. Sci. Technol. 15, 1300 (2002)

Open Access This is an open access article distributed under the terms of the Creative Commons Attribution License (http://creativecommons.org/licenses/by/3.0), which permits unrestricted use, distribution, and reproduction in any medium, provided the original work is properly cited. 